\title{
Editorial
}

\section{Regime-switching in financial markets}

Journal of Asset Management (2011) 12, 309. doi:10.1057/jam.2011.32

This issue looks at a number of studies of regime-switching in financial markets; a hardy perennial of a research topic, much loved by academics and central bankers, but rather underused by practitioners. The reasons for this unpopularity are many. These reasons are not based on ignorance but on very real difficulties in implementation. Furthermore, the structure of these models sits uncomfortably with the assumptions made by conventional optimisers. Converting these regime structures into global stock selection models with universes of, say, 5000 stocks seems not to be currently feasible.

The first paper, by Bulla and co-authors, looks at a Markov-switching asset allocation model, which uses current information on volatility to adjust the market exposure. They show that, compared with buy-and-hold, there are increased returns and reduced volatility. The second paper, by Guidolin and $\mathrm{Ria}$, converts regime-switching in first and second moments into regime-switching in mean variance frontiers. The data used here are Morgan Stanley investable indices for five countries/regions. The third article, by Haque, provides a theoretical contribution, in that it generalises the famous Barberis and Shleifer model of style switching. It is known that this model leads to small order ARMA models in relative style returns and current research looks at this structure as a way of forecasting when the switch may occuThis paper, by focusing on leaders and followers, may help in forecasting style switching and lead to new implementations of dynamic style allocation. The final paper, by van Vliet and Blitz, looks at asset allocation across different phases in the business cycle and identifies four phases, expressible in terms of time variation and risk and returns.

Concluding this issue provides a thorough examination of current research ideas on regime-switching. A point that comes through three of the papers very clearly is that, if we can implement regime-switching successfully, it is going to be with respect to asset classes or a small number of factors. For those of our readers who may wish to apply these ideas to stock selection models, it is clear that the only way forward is to regime-switch some of the key factors in the model. If a typical model has as factors market indices, style variables and possibly statistical factors, then it is not clear as yet which of these should be the most important ones for regime-switching. This may be a good topic for future research.

Stephen Satchell Editor E-mail: ses999gb@yahoo.co.uk 